Final manuscript of published paper: Journal of Management \& Marketing in Healthcare. Vol. 2, Issue 3, August 2009, pp. $278-292$.

\title{
Deploying a spreadsheet tool for early economic value assessment of medical device innovations with healthcare decision makers
}

\author{
M. P. Craven, S. P. Morgan, J. A. Crowe, B. Lu
}

MATCH (Multidisciplinary Assessment of Technology Centre for Healthcare)

University of Nottingham, Dept. of Electrical and Electronic Engineering

University Park, Nottingham, NG7 2RD. match@nottingham.ac.uk http://www.nottingham.ac.uk/match

Keywords: medical device evaluation, innovation assessment, collaborative decision-making, health economics, cost effectiveness.

Authors: The authors are with the Nottingham hub of the Multidisciplinary Assessment of Technology Centre for Healthcare (MATCH). MATCH is an academic-industry-healthcare professional collaboration that was created in 2003 with core funding from the UK Engineering and Physical Sciences Research Council as an Innovative Manufacturing Research Centre. The centre and its associated research programme are based across four UK universities: Birmingham, Brunel, Nottingham and Ulster. In addition to the academic effort, MATCH is a partnership of subscribing businesses and organisations of the National Health Service with additional support for SMEs from the Department of Trade and Industry and Invest Northern Ireland. MATCH aims to support the healthcare technology sector and its user communities by creating methods to assess value from concept through to mature product. MATCH addresses three perspectives on medical devices: new methods to assess value employing economic modelling; decision-making tools to facilitate optimised processes for design and manufacturing; and methods to ensure better engagement with users over the entire development cycle. Amongst the current projects at the Nottingham hub, MATCH is working towards improvement of innovation and procurement processes for medical technologies. Dr. Michael Craven is a Senior Research Fellow. Dr. Steve Morgan and Dr. John Crowe are MATCH investigators and Nottingham hub co-directors. Dr. Bo Lu is a Research Fellow.

\begin{abstract}
Early stage evaluation of medical device innovations is important for healthcare decision-makers as much as for manufacturers, meaning that a wider application of a basic cost-effectiveness analysis is becoming necessary outside the usual expert base of health technology assessment specialists. Resulting from an academic-industry-healthcare professional collaboration, a spreadsheet tool is described that was designed to be accessible both to professionals in healthcare delivery organisations and to innovators in the healthcare technology industry who are non-experts in the field of health economics. The tool enables a basic cost-effectiveness analysis to be carried out, using a simplified decision-tree model to compare costs and patient benefit for a new device-related procedure with that of standard care employing an incumbent device or other alternative. Such a tool is useful to healthcare professionals because it enables them to rapidly elucidate the cost-effectiveness of heterogeneous innovations by means of the standard quality adjusted life year (QALY) measure of clinical outcome, which is intended to be broadly comparable across treatments. For the innovator or manufacturer it helps them focus on what is required for future stages of development, in order to fill gaps in the input data and so further strengthen their case from a health economics perspective. Results are presented of first experiences from deploying the tool on three medical device exemplars, in face-to-face meetings of the NHS National Innovation Centre (NIC) along with the innovator or clinical champion. The results show that mapping of device-related innovations to the tool is achievable in a short meeting
\end{abstract}


between the NIC and the innovator using expected costs, outcomes data from the literature and estimates of ranges for unknown input data. Whilst the result of a simplified analysis is not expected to be definitive, the process of reasoning is found to be illuminating for the parties involved, enabling innovators to articulate the benefits of their innovations and for all parties to highlight gaps in data and evidence that will be required to take the innovation forward. The partnership model of the authors' organisation supports the kind of cooperative design approach that is necessary to produce the kind of tool described.

\section{Introduction}

Economic evaluation is commonplace in the assessment of therapies involving drugs and medical devices. In the UK, in the National Health Service (NHS) context, a cost-effectiveness analysis (CEA) is an important component of health technology assessments of treatments that are carried out for organisations such as the National Institute for Health and Clinical Excellence (NICE) of England and Wales, Quality Improvement Scotland (QIS) and the Department of Health, Social Services and Public Safety Northern Ireland (DHSSPNI). The need for increased capacity in the NHS to assess value through consideration of both costs and benefits is growing. At the local level, primary and acute hospital trusts are already tasked to demonstrate the quality of care through value indicators ${ }^{1}$. Acting on the outcomes of the Healthcare Industries Task Force (HITF) ${ }^{2,3}$, the Purchasing and Supply Agency (PASA) is now carrying out its own reviews and analysis of economic evidence of products through the Centre for Evidence-based Purchasing (CEP). Furthermore, the launch of the National Innovation Centre (NIC), another HITF initiative, has made pertinent the need for accessible tools to enable decision-makers to assess new healthcare technologies that are disclosed to them. The NIC aims to speed up the development of pre-commercial technologies likely to benefit the NHS, and has already produced a set of web-based tools to help innovators. In particular, the NIC's Scorecard tool ${ }^{4}$ provides an automatically generated self-assessment and also allows the innovation to be submitted for review by NIC experts if necessary. Scorecard is therefore a gateway to the innovation's detailed assessment, such that if a submission meets an NIC priority, an innovator may be offered a deeper due diligence service (covering IP, legal, commercial and financial aspects) with the aim of building a full business case. As a measure of the take-up of such tools, in August 2008 the NHS National Innovation Centre had received 151 submissions of an idea to its Scorecard tool and 500 out of the 939 registered individuals had used it for their own use without submitting it to NIC.

Resulting from this focus on accelerating the adoption of innovations into the NHS, technology businesses that are developing new medical devices for sale in the NHS are being encouraged to think about cost-effectiveness analysis at an ever earlier stage in the innovation process. To assist with this, the UK-based research programme (Multidisciplinary Assessment of Technology Centre for Healthcare $(\mathrm{MATCH})^{5}$ is collaborating with the NIC to develop and validate a new tool that is intended to be suitable for non-expert health economists to carry out, with minimal assistance, a basic costeffectiveness assessment of their innovation. The prototype tool was developed within the MATCH programme and has recently been made available to all its partners with an expectation for wider dissemination. The tool we describe is aimed at early stage cost-effectiveness which would ideally be applied after the NIC's Scorecard gateway. The following sections will describe the function of the tool, and present the results of research with three examples where it has been used to determine an estimate of cost effectiveness during meetings of MATCH and the NIC together with the participation of the innovator or clinical champion.

\section{Rationale}


The MATCH programme, which is now in its sixth year since its inception in 2003, has one of its major objectives the development and dissemination of methods and tools to support device manufacturers and health providers in their assessment of value of innovations in medical technologies. Health economics methods, related to the practice of Health Technology Assessment (HTA), are central to this approach. Early on in its research, MATCH conducted detailed interviewed with its industry partner base (12 interviews over a period of 10 months from March 2004 to January 2005) where each was asked about its approach to product development together with a set of questions focused on development of a specified product with reference to MATCH's research themes. This enabled MATCH to discover which kinds of decision aids they were using. Few had used health economics and none amongst SMEs and start-ups. Subsequently, at MATCH partner conferences, and through focussed seminar events to a wider audience of industry and health providers, it became clear that greater exposure to HTA methods was needed. Furthermore, as MATCH grew its relationship with the UK National Health Service, it became clear that translation of HTA methodology of NICE into a decision aid for estimating cost-effectiveness of specific medical device innovations would be useful. Out of these relationships emerged the idea for a software tool which is introduced in the following sections.

\section{NICE 'reference case' for economic evaluations}

There are a number of different methods of health economic evaluation that are typically used to assess drugs and devices as described by Drummond et al $^{6}$. In order to create consistency in decision making between alternatives (where a treatment is assessed against one or more comparators), NICE has adopted a 'reference case' approach to economic evaluation that has as its basis a cost-utility analysis (CUA). A CUA employs the Quality Adjusted Life Year (QALY) as a measure of the health benefits. This reference case can be summarised as shown in Table $1^{7}$.

Table 1 Summary of National Institute for Health and Clinical Excellence (NICE) reference case ${ }^{7}$.

\begin{tabular}{|c|c|}
\hline $\begin{array}{l}\text { Element of health technology } \\
\text { assessment }\end{array}$ & Reference case \\
\hline Defining the decision problem & The scope developed by the Institute \\
\hline Comparator & Alternative therapies routinely used in the National Health System (NHS) \\
\hline Perspective on costs & NHS and Personal Social Services (PSS) \\
\hline Perspective on outcomes & All health effects on individuals \\
\hline Types of economic evaluation & Cost-effectiveness analysis \\
\hline Synthesis of evidence on outcomes & Based on a systematic review \\
\hline Measure of health benefits & Quality-adjusted Life Years (QALYs) \\
\hline $\begin{array}{l}\text { Description of health states for } \\
\text { calculation of QALYs }\end{array}$ & Health states described using a standardised and validated generic instrument \\
\hline $\begin{array}{l}\text { Methods of preference elicitation for } \\
\text { health state valuation }\end{array}$ & $\begin{array}{l}\text { Choice-based method, for example, time trade-off, standard gamble (not rating } \\
\text { scale) }\end{array}$ \\
\hline Source of preference data & Representative sample of the public \\
\hline Discount rate & An annual rate of $3.5 \%$ on both costs and health effects \\
\hline Equity provision & $\begin{array}{l}\text { An additional QALY has the same weight regardless of the other characteristics of } \\
\text { the individuals receiving the health benefit }\end{array}$ \\
\hline
\end{tabular}


In addition a sensitivity analysis is recommended to examine to effect of uncertainty in input data. It can be seen that the NICE reference case is quite prescriptive, requires expertise to apply it comprehensively and will take a significant amount of time to apply. Therefore, in producing its early stage tool for use by medical device companies and the National Innovation Centre, MATCH needed to ask:

What features of the NICE reference case are important for an early stage analysis?

$\circ$ What kind of tool is most readily accessible to businesses and healthcare professionals who may have little prior knowledge of health economics?

- What features of a tool are required to map the range of innovations presented to the NIC?

- Will the tool enable an initial assessment to be made in a short time period?

$\circ$ Will the results of the analysis focus the innovators in the right direction?

From the detailed interviews with MATCH's partners it was understood that those involved in product development decision processes were familiar with basic 'office' tools (word processors and spreadsheets) and also that those few that had performed health economics evaluations had used Microsoft Excel. It was therefore decided to use the Excel spreadsheet as the basis for a simple tool to model cost-utility analyses, with an emphasis on providing graphical output and an element of sensitivity analysis. Furthermore the tool would implement the simplest decision model possible with a minimum of input variables, to reduce the time and effort to populate the model whilst maintaining features that would enable a meaningful comparison to be made. The initial proposal for producing an Excel-based tool was made in 2005. The tool was subsequently designed in consultation with health economists in MATCH and through listening to participants of MATCH presentations at industry events who included manufacturers, health providers, business development executives and technologies transfer specialists, where we outlined and discussed health economics methods with them. Hands-on experience in producing simple early-stage health economic models alongside industry partners had been gained in MATCH through managed projects, which also helped inform the initial design. The tool was tested and refined incrementally through its use in educational workshops with both industry and NHS partners, from observation and discussion with its initial users and from questionnaire feedback at those events. The design and refinement process took place late-2005 to early-2007. Use of the tool to support the NHS in assessing innovations, as described in the rest of this paper, was managed as a Research Partner project with the National Innovation Centre as a component of their subscription to MATCH, whereby a set of innovation exemplars was selected by the NIC and agreed. This exemplar project work took place between May and November 2007.

\section{Cost utility analysis}

Taking as a comparator the alternative treatment against which an innovation is to be judged, a CUA models both the difference in costs and the difference in health effects, where health effects are measured in QALYs. Once determined, it is possible to plot the results on a 'cost-effectiveness plane' as illustrated in Figure 1. The plane is divided into four quadrants which illustrate the four possible results of such an analysis:

I New treatment is more effective and more expensive

II New treatment is more effective and less expensive

III New treatment is less effective and less expensive IV New treatment is less effective and more expensive 
The result of any particular comparison is plotted as a single point on the plane. Four points are shown on the graph to illustrate hypothetical cases A, B, C and D where the innovation is being compared against the incumbent treatment.

Ideally the innovation in question will be both cheaper and more clinically effective than the incumbent (case A) so that in principle it has a very good chance of being adopted. However, a more expensive innovation may also be considered cost-effective (case B) if there is a health gain which puts the innovation beneath the willingness-to-pay threshold (WTP) of the payer, as illustrated by the dotted line (where the slope of this line is in units of $£$ per QALY). A measure of the effectiveness of the innovation is then gained by comparing this threshold with the Incremental Cost Effectiveness Ratio (ICER) which is defined as:

$$
I C E R=\frac{\text { Difference } \text { in costs of treatment }(\Delta £)}{\text { Difference in health effects }(\triangle Q A L Y S)}
$$

A new treatment is likely to be deemed cost effective by the payer if the ICER is less than the WTP threshold. For case $\mathrm{C}$ the point is above the WTP threshold, and so the innovation is not likely to be considered cost effective. Case D illustrates the problem of uncertainty, where cost-effectiveness may be sensitive to parameters in the model, some more than others. In this case a too high selling price set by the manufacturer could affect its viability if that price took it over the WTP threshold. Clearly it will be important to examine the effects of other parameters on the location of the point on the costeffectiveness plane. It is worth noting that another way of considering the cost-effectiveness from the manufacturer perspective is to see whether there is enough headroom between the price of the device and the WTP threshold to ensure that the innovation is profitable ${ }^{8}$.

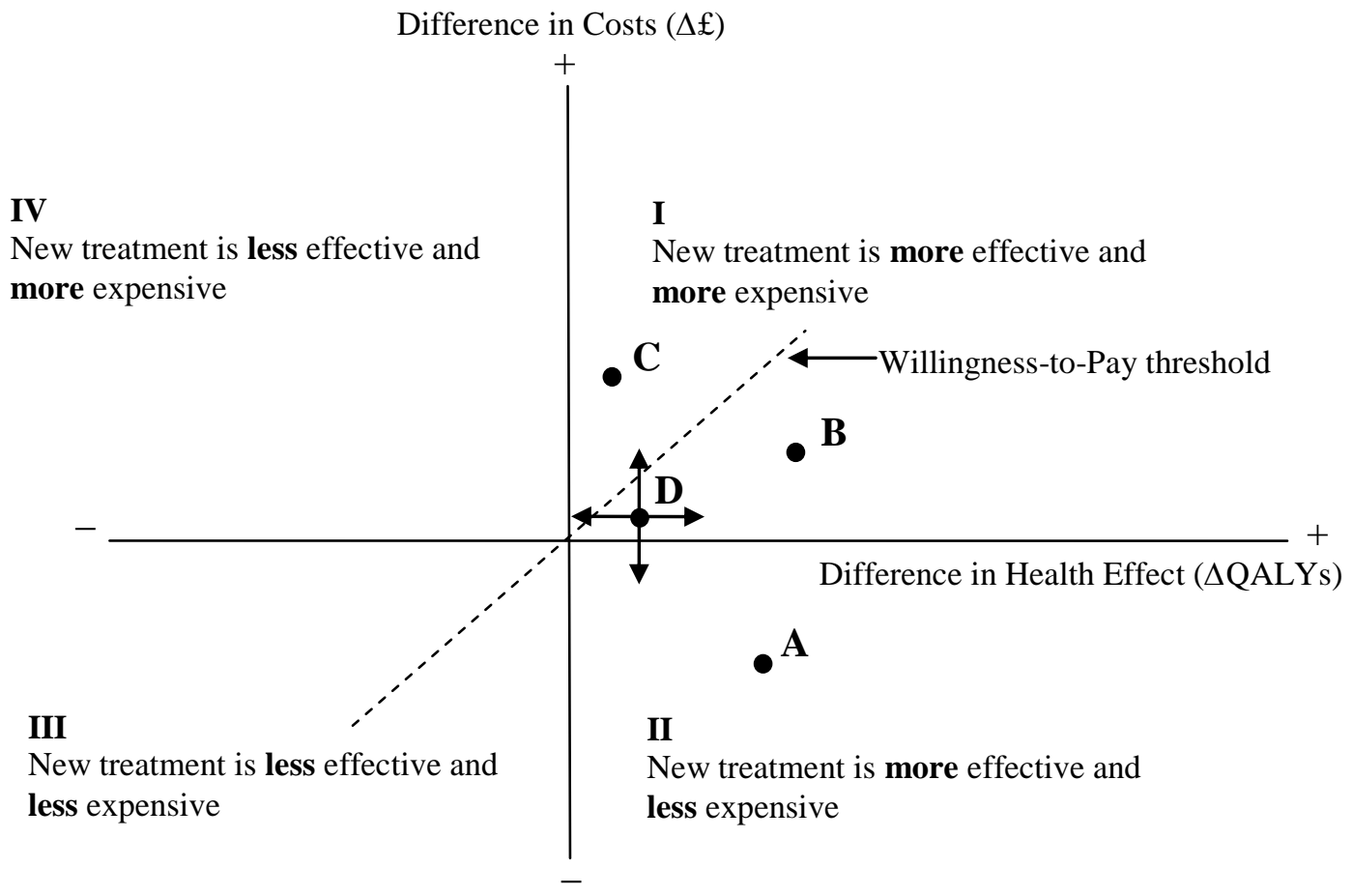

Figure 1: The Cost Effectiveness Plane 


\section{Basic decision tree model}

As a baseline for a simplified early stage model, we notice that modelling is often required for diagnosis and treatment of an acute illness where the costs are measured up to a one year time horizon. For short term treatments of this kind, cost discounting is not required. QALY data can be obtained from the literature, or estimated from published utility values, typically obtained from EuroQoL EQ-5D measures ${ }^{9}$, or similar measures such as Health Utilities Index HUI ${ }^{10}$. If it is necessary to apply discounting, this is done according to the NICE reference case which currently stipulates a discount rate of 3.5\% for both costs and QALYs. For chronic conditions a typical approach is to annualise costs, and then apply discounting. If the costs and QALYs can be adequately determined, all that remains to be found are the probabilities of a patient being treated with the innovation vs. the incumbent, and the probabilities of the possible outcomes of the treatment or non-treatment (either 'healthy' or 'nothealthy') so that this data can then be used to populate a decision tree, as shown in Figure 2.

For each branch of the decision tree the costs and probabilities are combined to obtain the overall costs for the innovation (C1) and for the incumbent (C2). Similarly, utilities and probabilities are combined over the appropriate time horizon in order to give QALY measures for the innovation (Q1) and incumbent $(\mathrm{Q} 2)$, therefore allowing the ICER to be calculated as $(\mathrm{C} 1-\mathrm{C} 2) /(\mathrm{Q} 1-\mathrm{Q} 2)$. In the hypothetical example of a comparison shown in figure 2 difference in cost is $-£ 4,745$ and the difference in QALYs is 0.84 , so the ICER is $-£ 5,668.95$ per QALY which represents a saving to the NHS for the innovation being modelled as well as a health gain. Uncertainty in the ICER can be calculated using the Solver component of Excel to find its overall maximum and minimum over the range of multivariate inputs (an alternative, but more involved technique is Monte Carlo analysis).

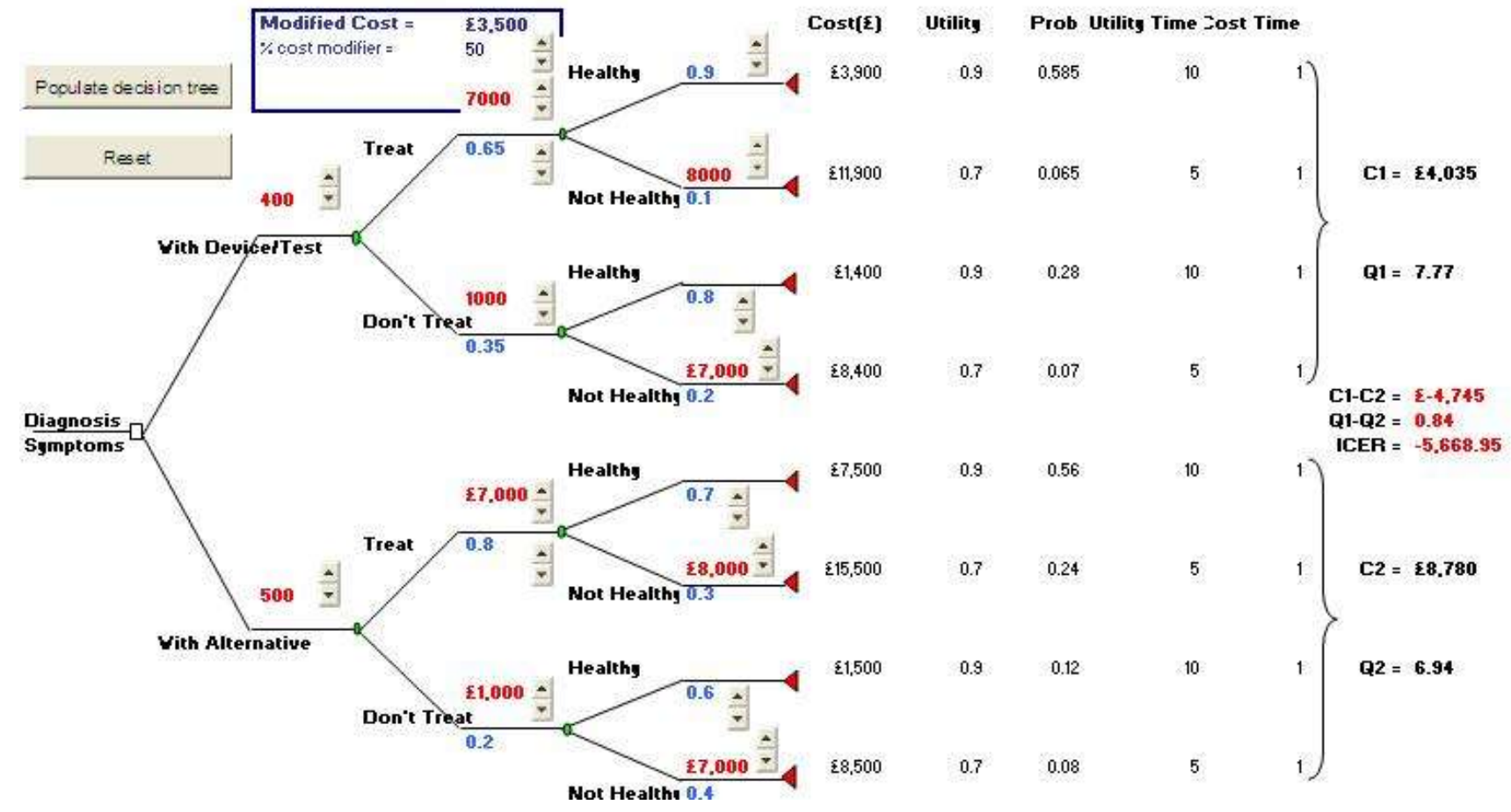

Figure 2 Basic decision tree model for a Cost Utility Analysis (from Excel spreadsheet chart) 


\section{The Excel spreadsheet tool}

A number of software packages exist to perform decision-tree analysis e.g. TreeAge. However, since many professionals are familiar with office tools, as we confirmed in our partner interviews, we aimed to reduce the learning curve for early stage cost-utility modelling using a set of Microsoft Excel spreadsheets. The features included as individual sheets were:

1. Instructions

2. Data Input (Figure 3)

3. Interactive decision tree (Figure 2)

4. Cost-effectiveness plane chart (Figure 4)

5. Financials (Figure 5)

Instructions are provided 'in package' to enable quick learning of the spreadsheet features and a separate user guide document are also provided. For the data input sheet (Figure 3 ) each parameter is entered as a value between a range of possible minimum and maximum values which are also specified. To assist with sensitivity analysis, a feature was included whereby the tool's user can select up to 4 parameters (via the tick boxes shown in figure 3 ) whose values can later be varied on the costeffectiveness chart (Figure 4) between their maximum and minimum values and so judge their sensitivity effects on the ICER.

The interactive decision tree (shown previously in Figure 2) is populated with the values from the Data Input sheet (Figure 3) and on this sheet the user can immediately see the resulting ICER and also examine the effect of varying costs and probabilities within their specified ranges. The decision tree is essentially a text book health economic model but with the addition of one novel feature that gives an extra degree of flexibility. This feature is a percentage cost modifier which allows the modeller to estimate an efficiency gain for the innovation, which makes the 'standard' treatment cheaper by a percentage.

The cost-effectiveness chart (Figure 4) displays the ICER as a single point and the ranges of values between maximum and minimum of the cost difference and QALY differences are shown as vertical and horizontal bars. It is widely accepted that NICE has an adopted cap of $£ 20,000$ to $£ 30,000$ per quality adjusted life year (QALY) gained, although it does not accept or reject healthcare technologies on cost effectiveness grounds alone and there are examples of accepted treatments above this range ${ }^{11,12}$. For this reason we include a selectable cap so that $£$ per QALY thresholds above and below $£ 30,000$ can be plotted, which includes zero to illustrate cost minimisation. Since the range of the ICER depends on multiple costs and utilities from the input data, the 'Solver' add-in feature of Excel is used to find the maximum and minimum values.

Finally, resulting from discussion with industrial partners a break-even analysis sheet (Figure 5) is included, where the return on investment can be calculated, given the selling price (or reimbursement), a predicted market share, unit manufacturing cost, and non-recoverable expense. For the example shown in Figure 5 the innovation provides profit for the company after sales of 5714 units, which includes fixed development costs and variable manufacturing cost per unit. 


\begin{tabular}{|c|c|c|c|c|}
\hline Variable & Description & value & Min & $\max$ \\
\hline \multicolumn{5}{|l|}{ Costs $(£)$} \\
\hline $\mathrm{Ct}$ & Cost of treatment (not including cost of device/test) & 7000 & 5000 & 10000 \\
\hline $\mathrm{Ct}^{\prime}$ & Cost of not treating (or 'do nothing') & 1000 & 500 & 1500 \\
\hline Cth' & Cost of further treatment after failed first treatment & 8000 & 5000 & 10000 \\
\hline C1d & Cost of device/test (not including cost of treatment) & 400 & 200 & 30000 \\
\hline $\mathrm{C} 2 \mathrm{~d}$ & Cost of alternative (not including cost of treatment) & 500 & 200 & 30000 \\
\hline$\alpha$ & Cost modifier for treatment with device/test & 50 & 0 & 150 \\
\hline \multicolumn{5}{|c|}{ Probabilities (\%) } \\
\hline P1t & Probability of treating with device/test & 65 & 60 & 90 \\
\hline P2t & Probability of treating with alternative & 80 & 60 & 90 \\
\hline P1th & Probability of healthy outcome after treating with device/test & 90 & 85 & 95 \\
\hline P1t'h & Probability of healthy outcome after not treating with device/test & 80 & 75 & 85 \\
\hline P2th & Probability of healthy outcome after treating with alternative & 70 & 65 & 75 \\
\hline P2t'h & Probability of healthy outcome after not treating with alternative & 60 & 55 & 65 \\
\hline \multicolumn{5}{|l|}{ Utilities } \\
\hline U1th & Utility of Treated with device/test (Healthy) & 0.90 & 0.80 & 1.00 \\
\hline U1th' & Utility of Treated with device/test (unHealthy) & 0.70 & 0.60 & 0.80 \\
\hline U1t'h & Utility of not Treated with device/test (Healthy) & 0.90 & 0.80 & 1.00 \\
\hline U1t'h' & Utility of not Treated with device/test (unHealthy) & 0.70 & 0.60 & 0.80 \\
\hline U2th & Utility of Treated with alternative (Healthy) & 0.90 & 0.80 & 1.00 \\
\hline U2th' & Utility of Treated with alternative (unHealthy) & 0.70 & 0.60 & 0.80 \\
\hline U2t'h & Utility of not Treated with alternative (Healthy) & 0.90 & 0.80 & 1.00 \\
\hline U2t'h' & Utility of not Treated with alternative (unHealthy) & 0.70 & 0.60 & 0.80 \\
\hline \multicolumn{5}{|c|}{ Time Horizon for Utility (year) } \\
\hline TU1th & Horizon of life of Treated with device/test (Healthy) & 10 & 1 & 10 \\
\hline TU1th' & Horizon of life of Treated with device/test (unHealthy) & 5 & 1 & 10 \\
\hline TU1t'h & Horizon of life of not Treated with device/test (Healthy) & 10 & 1 & 10 \\
\hline TU1t'h' & Horizon of life of not Treated with device/test (unHealthy) & 5 & 1 & 10 \\
\hline TU2th & Horizon of life of Treated with alternative (Healthy) & 10 & 1 & 10 \\
\hline TU2th' & Horizon of life of Treated with alternative (unHealthy) & 5 & 1 & 10 \\
\hline TU2t'h & Horizon of life of not Treated with alternative (Healthy) & 10 & 1 & 10 \\
\hline TU2t'h' & Horizon of life of not Treated with alternative (unHealthy) & 5 & 1 & 10 \\
\hline \multicolumn{5}{|c|}{ Time Horizon for Cost (year) } \\
\hline TC1th & Horizon of cost of Treated with device/test (Healthy) & 1 & 1 & 1 \\
\hline TC1th' & Horizon of cost of Treated with device/test (unHealthy) & 1 & 1 & 1 \\
\hline TC1t'h & Horizon of cost of not Treated with device/test (Healthy) & 1 & 1 & 1 \\
\hline TC1t'h' & Horizon of cost of not Treated with device/test (unHealthy) & 1 & 1 & 1 \\
\hline TC2th & Horizon of cost of Treated with alternative (Healthy) & 1 & 1 & 1 \\
\hline TC2th' & Horizon of cost of Treated with alternative (unHealthy) & 1 & 1 & 1 \\
\hline TC2t'h & Horizon of cost of not Treated with alternative (Healthy) & 1 & 1 & 1 \\
\hline TC2t'h' & Horizon of cost of not Treated with alternative (unHealthy) & 1 & 1 & 1 \\
\hline \multicolumn{5}{|c|}{ Discount rate (\%) } \\
\hline $\mathrm{D}$ & Discount rate & 3.5 & 0 & 6 \\
\hline
\end{tabular}

Figure 3 Data input for basic decision tree model (from Excel spreadsheet) 


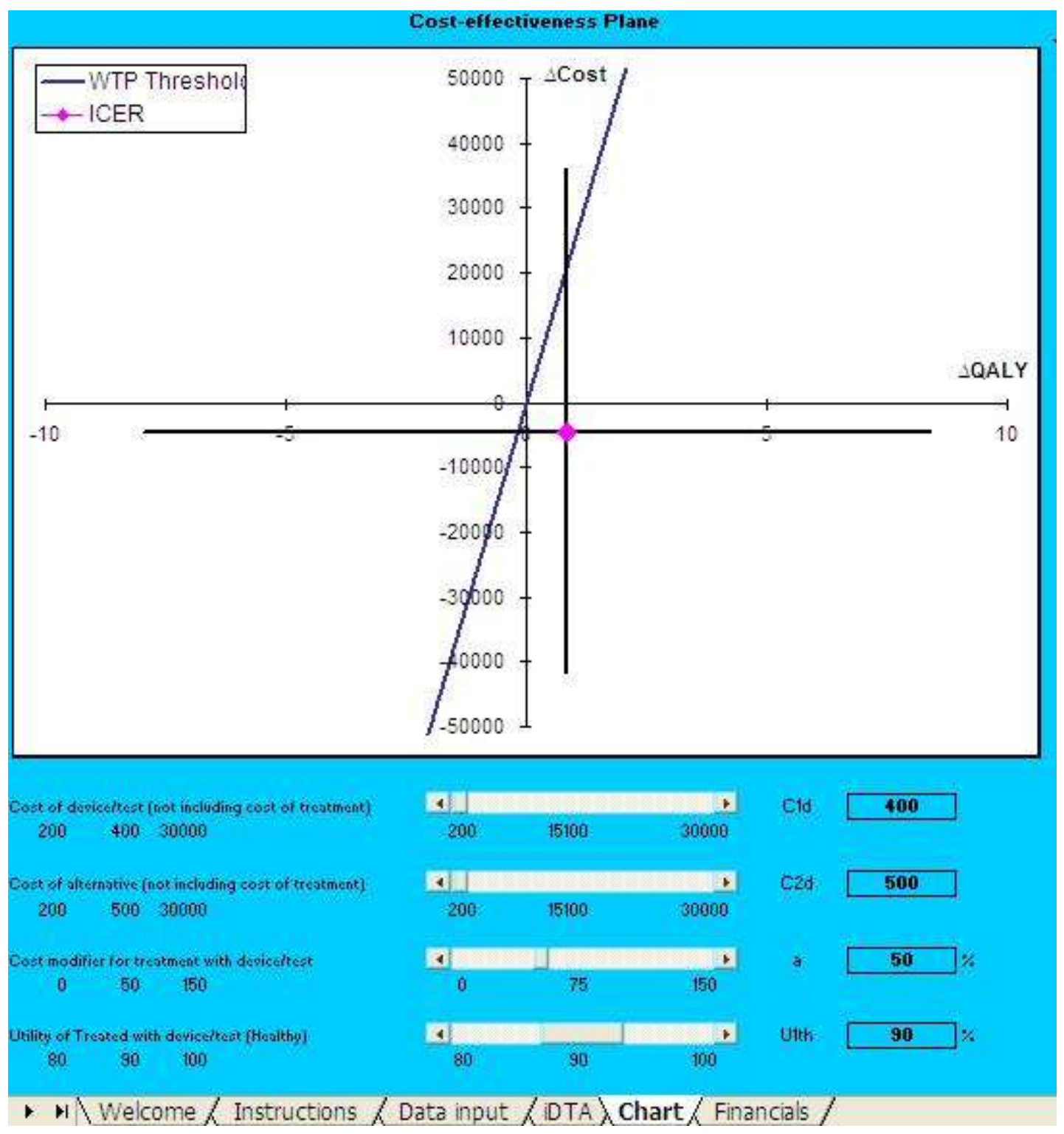

Figure 4 Cost-effectiveness chart with scroll bars allowing ICER sensitivity to selected input data to be examined (from Excel spreadsheet) 


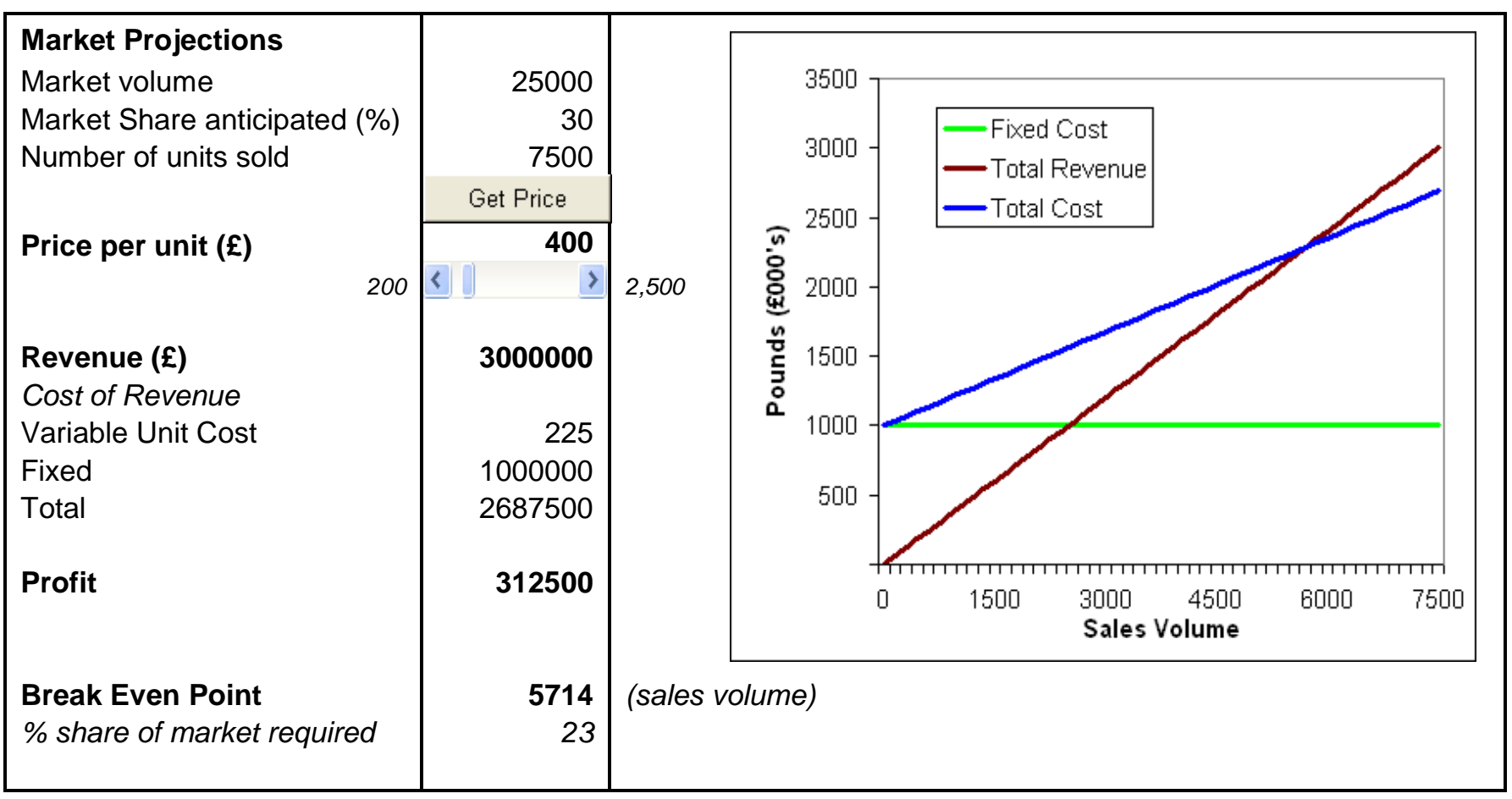

Figure 5 Break even analysis (from Excel spreadsheet, Financials tab)

\section{Exemplar studies}

In order to investigate the potential of the tool, three exemplars were chosen to be conducted in collaboration with the NIC and the innovator or clinical champion of the medical device. All of the exemplars are part of treatment for diseases that are prevalent in the UK. In the following, for reasons of commercial confidentiality it is not the intention either to describe the innovation in detail or specify device costs, nor to provide an answer for cost-effectiveness or discuss the validity of results, but rather to describe the experience of using the tool.

\section{1) Saturation driven oxygen therapy (SDOT)}

Oxygen therapy involves providing patients with a prescribed flow of oxygen from a cylinder or concentrator. It is a standard treatment for patients with respiratory conditions, such as emphysema, cystic fibrosis and others, providing improvement in cardiac function, exercise ability and quality of life. However, the existing treatment is not responsive to variations in the patient's oxygen demand, resulting in periods of insufficient oxygenation that may accelerate disease progression and worsening cardiovascular health. Saturation Driven Oxygen Therapy (SDOT) is a system developed by a UK company that automatically adjusts the oxygen flow to match the patient's measured demand. It is fitted to an existing oxygen delivery system. The flow control system is intended to improve health by avoiding periods of insufficient oxygenation, however it also reduces demands on GPs for setting prescription levels, and users have suggested it reduces patient anxiety about home treatment. SDOT is also expected to reduce the time required for assessing a patient under NICE guidelines, since some of the time of consultation is taken up with a series 'shuttle walks' where the patient is asked to walk between two points and oxygen saturation is measured using a pulse oximeter. It may take 30-40 minutes of shuttle walks before an average oxygen flow setting is determined, whereas the SDOT 
system, based on continuous pulse oximeter measurement, should assist with the setting and then maintain the correct flow automatically.

The costs and benefits of SDOT were modelled using the decision tree spreadsheet during a 3 hour session at the NIC in consultation with the innovator. Some costs and probabilities were available from recent clinical trials, and a utility value for a patient with emphysema from a published reference on chronic conditions was used as the baseline for health benefits. The innovation requires an additional annual cost on top of the existing therapy that is represented in the first branch level of the upper 'innovation' decision tree. The added cost in the first branch of the lower 'incumbent' tree, for conventional oxygen therapy, is zero. In the second level, a combined consultation, assessment and treatment cost is entered, and cost of non-treatment (consultation only) is also entered. An appropriate cost modifier was entered for SDOT based on an assumption that the assessment time is reduced because it is not necessary to spend time finding the correct oxygen level for the patient, as discussed above. Finally a 'not healthy' cost was entered to account for the possibility of A\&E admission due to exacerbation or disease progression. Estimates of probabilities and utilities were entered using the baseline emphysema utility value modified by reasoning about the health benefits of SDOT (e.g. reduction in periods of excessive/insufficient blood oxygen).

From the values entered, one is able to infer that the additional cost of SDOT may be balanced by the benefits. By running the Solver component of the Excel spreadsheet for appropriate input ranges of costs, probabilities and utilities, it was revealed that overall cost differences between SDOT and conventional oxygen therapy vary to the extent that in the very worst case, the ICER could possibly go over a WTP threshold of $£ 30,000$ per QALY. However this would only be for substantially higher device and treatment costs, lower costs of dealing with failed treatments, and the smallest QALY gain. In the best case, using SDOT could result in a substantial cost saving for a modest improvement in clinical benefit. Since many of the values are estimates, it is important to stress once again that it is not the precise value of the ICER that is important, but rather the reasoning involved in mapping the problem to the simplified tree and from the process of examining the uncertainties.

According the innovator. the main questions identified by the modelling SDOT in the tool were:

Q1. What range of consultant and GP prescribing time, where saved by use of SDOT, justify value for money when used as a diagnostic device?

Q2. Can sufficiently accurate utility values be obtained from the literature to justify the model's claim for improved benefit, or what trial design will support this?

Q3. What are the additional costs to the NHS associated with non-optimal delivery of oxygen therapy, and the true probability of A\&E admission following poor quality home treatment?

Answers to these questions informed further development and evaluation of the device, and also identified new market opportunities for the company.

\section{2) Optical blood glucose monitoring}

The importance of good control of blood glucose level in diabetes is well known and this is most commonly achieved by taking a blood sample from the finger using a lancet (finger stick) and then measuring it on a test strip with a blood glucose meter. One problem for the user associated with the technique is that it is invasive. Repeated perforation of the finger (or alternative test site) causes skin 
and nerve damage and the process is not pain free. For this and a variety of other reasons, many patients do not test themselves as often as recommended. By removing the need to sample blood directly, the innovation of non-invasive blood glucose metering aims to overcome these problems. Such a device is being developed by a UK company, using an optical technique that measures blood glucose level in the eye.

As for the first exemplar, the innovation was compared with a conventional treatment, in this case using lancet, test strip and blood glucose meter as the incumbent. In the case of the comparator the meter itself is generally provided free of charge by the manufacturer and the annual per patient cost is the price of the consumables (lancets and test strips) estimated as an annual cost averaged over all patients. The cost of the new meter was entered as a modification of the annual consumables cost. It was not necessary to include the cost of insulin therapy resulting from testing on the basis that this is arguably the same for both arms of the comparison (dependent on proportion of Type 1 and Type 2 diabetics and degree of insulin use). An average cost of treating the complications of diabetes was entered. The probabilities were estimated such that the probability of healthy outcome was greater for the innovation, due to increased compliance. A baseline utility for a patient with diabetes mellitus was obtained from the literature. A decision tree model resulted from this mapping, much of the meeting time being taken in discussing the utility values and probabilities, and how utilities change with disease progression.

In this example, it was assumed that the main economic case for an optical meter rests on the annual cost saving on consumables and improved compliance that results in a higher proportion of healthy patients. As for the first exemplar, it will be necessary to fully justify the input data values chosen, and apply discounting so that the 'not healthy' costs and QALYs are compounded into their present values, since the expected health gains from better diabetes management will not all be realised in the short term.

\section{3) Varicose vein closure techniques}

There are a number of techniques for varicose vein treatment using endovenous ablation to close the veins by chemical, laser or radio-frequency (RF) means. As well as the choice of technique, treatments vary as to whether the patient undergoes local anaesthesia (LA) or general anaesthesia (GA) and whether one or two legs are treated in the same session (unilateral or bilateral). These alternative endovenous treatments are currently undergoing close scrutiny in the UK with the NIC, CEP and HTA Programme all involved with evaluation. The potential for cost saving, together with higher throughput by delivery outside of secondary care, is of particular interest for the NHS 18 Week Programme.

For the purposes of modelling with a health economics tool, we can consider comparison of several patient pathways. Two such pathways were determined in a meeting with a surgeon who was carrying out clinical research into the alternative endovenous treatments. Firstly, it is necessary to model the cost effectiveness of unilateral varicose veins therapy carried out under LA using laser or RF, but with a probability of some patients requiring a second treatment. According to the surgeon who participated in the discussions with NIC, a proportion of his eligible patients elect for treatment under LA which is quicker that with GA. However therapy may be painful from heating of tissue and if vein-stripping is to be carried out in addition to the endovenous treatment, so this could limit the amount of vein treated in a single treatment session if carried out under LA.. Secondly it is necessary to model the cost effectiveness model of unilateral varicose vein therapy performed using endovenous ablation under GA. A different proportion of patients prefer this option or are best served by it for anatomical reasons. This provision requires several essential staff in theatre and recovery. In both cases the incumbent 
treatment can be vein stripping or chemical sclerotherapy. The meeting was concluded with a commitment to obtain further cost data in order to fully populate the decision trees. Even though the model was not fully completed during the meeting between NIC and the surgeon, the framing of discussions in terms of decision trees was instrumental in increasing understanding of the NIC about the alternatives and factors impacting on cost-effectiveness. Subsequent discussions with the manufacturer of the RF system also yielded some differences of option within the surgeon community with respect to treatment under LA versus GA. The majority of Trusts using this particular system are already using LA, including bilateral treatments, and most are moving to an outpatient setting. This subsequent dialogue also increased understanding by NIC of differences between the laser and RF alternatives.

\section{Conclusions}

With an increasing need to identify additional spending with metrics of patient benefit, the wider application of basic cost-effectiveness analysis has become necessary outside the usual expert base of health technology assessment specialists. In the context of the findings of the UK Health Industries Task Force, that a better assessment of value is required to bring new health technologies into the National Health Service, the tool described is seen to be facilitating a common understanding of value between healthcare decision-makers and medical device developers or clinician 'champions' who are amongst the first adopters of newly marketed devices.

Further exemplar work is needed to examine the generalisability of the tool in terms of number and depth of decision tree branches, balanced with a requirement to keep the model simple for non-expert use, and in the knowledge that data for a more complex model will be limited at the early stage of an innovation's development or deployment. As stated repeatedly, the tool is not intended to replace the need for a full NICE appraisal using a more complete data set, accepting that limited data will be the norm for early stage decisions. However more work needs to be done to examine the sensitivity to estimated data ranges and especially to research the effect on decisions from the making of optimistic or pessimistic estimates.

Conducting these exemplar studies with the tool on real users during its development prompted discussion about the 'percentage cost modifier parameter'. The rationale for inclusion of the feature was based on an example of a diagnostic device designed to assess burns by means of blood flow measurement, where the treatment is a skin graft. If the innovation resulted in better assessment of the area in need of grafting, it was argued that this could be modelled as an efficiency saving in the treatment and therefore cost of treatment could be reduced accordingly. However, for general use it is perhaps too tempting to use it as a 'fudge factor' to reduce the relative cost of the innovation. We have now decided to remove the feature and the user should enter cost savings to treatments directly.

A major lesson learnt from the exemplar work is that lack of hard data is not a barrier to mutual understanding of value at the early stage of development or deployment of a medical technology innovation. We also found that data may exist but not be readily available, which suggests that wider deployment of the tool would ideally be supported by an accessible repository of data for the purposes of health economics evaluation. Most importantly the tool was developed in close partnership with its users. The close academic-industry-healthcare professional linkage facilitated by MATCH supports and actively promotes this kind of cooperative design approach.

All of the focussed evaluation meetings were carried out over a half day period. However, some preparation is needed by all parties. Since conducting these exemplars, and after further consultations 
with partners in the medical devices industry, we have identified that an introduction to health economics, data sources and some hands-on training with the tool prior to the evaluation meeting would be beneficial. MATCH therefore decided to launch a series of tool workshops through the UKwide Medilink network which brings together medical manufacturing and distribution companies, hospitals and universities to stimulate innovation on a regional basis. This is taking place alongside a new phase of exemplar studies with both NIC and PASA.

\section{Acknowledgements and further information}

This paper was produced under the MATCH Programme (EPSRC Grant GR/S29874/01), although the views expressed are entirely those of the authors. We duly acknowledge Nick Botterill and Sukhvinder Johal, who although no longer with MATCH were also responsible for the initial design of the tool at the University of Nottingham, and MATCH researchers Lotte Steuten and Laura Vallejo-Torres from the Health Economics Research Group at Brunel University for their valuable advice. The authors also wish to acknowledge the ongoing collaboration of MATCH with the NHS National Innovation Centre through Brian Winn and Mary Green, who brought together the medical device innovators and NHS personnel who participated in the exemplar meetings. Please contact match@nottingham.ac.uk for information about obtaining the tool.

\section{References}

[1] NHS Institute for Innovation and Improvement, 'Better Care, Better Value Indicators', http://www.productivity.nhs.uk (accessed September 2008).

[2] Healthcare Industries Task Force (HITF) 'Better health through partnership: A programme for action', 17 November 2004, http://www.advisorybodies.doh.gov.uk/hitf/ (accessed August 2007). [3] Strategic Implementation Group (SIG), Healthcare Industries Taskforce, Innovation for health: making a difference, 6 March 2007, http://www.advisorybodies.doh.gov.uk/hitf/ (accessed August 2007).

[4] NHS National Innovation Centre, Scorecard, http://www.nic.nhs.uk/Scorecard/Pages/Scorecard.aspx (accessed October 2007)

[5] Craven M., Crowe J., Botterill N., Morgan S. and Williams H., MATCH (2004): A multidisciplinary approach to the advancement of Health Technology Assessment, Proceedings of the Institute of Physics and Engineering in Medicine (IPEM) 11th Annual Scientific Meeting, York, 6-8 September 2004, p;41, ISBN 1-9036-1323-X.

[6] Drummond M.F., Sculpher M.J., Torrance G.W., O’Brien B.J., Stoddart G.L. (2005) Methods for the economic evaluation of health care programmes, 3rd edn. Oxford University Press, Oxford.

[7] National Institute for Health and Clinical Excellence (2004), Guide to the Methods of Technology Appraisal, reference N0515, p;21. http://www.nice.org.uk/niceMedia/pdf/TAP_Methods.pdf (accessed October 2007).

[8] McAteer H. and Lilford R. (2008) 'The use of health economics in the early evaluation of regenerative medicine applications', European Cells and Materials, Vol. 16. Suppl. 3, p;5.

[9] The EuroQol Group (1990) 'EuroQol--a new facility for the measurement of health-related quality of life', Health Policy, Vol. 16, No. 3, December, pp;199-208.

[10] Horsman J., Furlong W., Feeny D., Torrance G. (2003) 'The Health Utilities Index (HUI®): concepts, measurement properties and applications'. Health Qual Life Outcomes, Vol. 1, No. 54. [11] Walker S., Palmer S. and Sculpher M.: The role of NICE technology appraisal in NHS rationing, British Medical Bulletin 2007, pp;1-14.

[12] Appleby J., Devlin N. and Parkin D. (2007) 'NICE's cost effectiveness threshold: How high should it be?', British Medical Journal, Vol. 335, 25 August, pp;358-359. 